\title{
Big Data in Manufacturing
}

\section{Matthew N. O. Sadiku1 ${ }^{*}$, Tolulope J. Ashaolu' ${ }^{2}$, Abayomi Ajayi-Majebi ${ }^{3}$, and Sarhan M. Musa ${ }^{1}$}

\author{
${ }^{1}$ Roy G. Perry College of Engineering, Prairie View A\&M University, Prairie View, TX, USA \\ ${ }^{2}$ College of Food Science, Southwest University, Tiansheng, Beibei, Chongqing, China \\ ${ }^{3}$ Department of Manufacturing Engineering, Central State University, Wilberforce, OH, USA
}

E-mail: sadiku@ieee.org; ashaolut@gmail.com; ajayi-majebi@centralstate.edu; smmusa @pvamu.edu

\author{
*corresponding author details: Professor Matthew N. O. Sadiku; sadiku@ieee.org
}

\begin{abstract}
In the data-driven economy, turning data into actionable analytics is the best way to boost efficiency, quality, and productivity. The manufacturing processes are getting more and more complex due to increasing demands. Manufacturers of all types of products are finding significant value in big data. The application of big data technologies in manufacturing sector is a relatively new interdisciplinary research area which incorporates automation, engineering, and data analytics. This paper provides an introduction on the use of big data in manufacturing.
\end{abstract}

Keywords: big data; data analytics; manufacturing

\section{INTRODUCTION}

Big data is everywhere and the big data revolution is upon us. Data has been helping businesses make better decisions for centuries. As shown in Figure 1, businesses from all types of industries have greatly benefited by adopting big data solutions [1]. This is true of healthcare, energy, finance, telecommunication, marketing, and sports. The manufacturing industry has joined the big data bandwagon as well. The manufacturing industry adds a lot of value to any nation's economy. Manufacturing is critical to national economies by providing jobs and improving innovation. It is well known as the most challenging and complex industry as far as variety of the products are concerned. There are different types of manufacturing including biopharmaceutical manufacturing, chemical manufacturing, discrete manufacturing, advanced manufacturing, smart manufacturing, cloud manufacturing, offshore manufacturing, lean manufacturing, green manufacturing, distributed manufacturing, predictive manufacturing, and computeraided manufacturing. Big data has been a fast-changing research area with many new opportunities for applications in manufacturing. Big data analytics can provide the manufacturing industry the need to succeed in an increasingly complex environment.

\section{BIG DATA CHARACTERISTICS}

Big data (BD) is a relatively newer technology that can help the food industry. The three main sources of big data are machines, people, and companies. Big data can be described with 42 Vs [2]. The first five Vs are volume, velocity, variety, veracity, and value [3].

\section{- Volume}

This refers to the size of the data being generated both inside and outside organizations and is increasing annually. Some regard big data as data over one petabyte in volume.

\section{- Velocity}

This depicts the unprecedented speed at which data are generated by Internet users, mobile users, social media, etc.
Data are generated and processed in a fast way to extract useful, relevant information. Big data could be analyzed in real time, and it has movement and velocity.

\section{- Variety}

This refers to the data types since big data may originate from heterogeneous sources and is in different formats (e.g., videos, images, audio, text, logs). BD comprises of structured, semi-structured or unstructured data.

\section{- Veracity}

By this, we mean the truthfulness of data, i.e. weather the data comes from a reputable, trustworthy, authentic, and accountable source. It suggests the inconsistency in the quality of different sources of big data. The data may not be $100 \%$ correct.

\section{- Value}

This is the most important aspect of the big data. It is the desired outcome of big data processing. It refers to the process of discovering hidden values from large datasets. It denotes the value derived from the analysis of the existing data. If one cannot extract some business value from the data, there is no use managing and storing it.

On this basis, small data can be regarded as having low volume, low velocity, low variety, low veracity, and low value. Additional five Vs has been added [4]:

\section{- Validity}

This refers to the accuracy and correctness of data. It also indicates how up to date it is.

\section{- Viability}

This identifies the relevancy of data for each use case. Relevancy of data is required to maintain the desired and accurate outcome through analytical and predictive measures. 


\section{- Volatility}

Since data are generated and change at a rapid rate, volatility determines how quickly data change.

\section{- Vulnerability}

The vulnerability of data is essential because privacy and security are of utmost importance for personal data.

\section{- Visualization}

Data needs to be presented unambiguously and attractively to the user. Proper visualization of large and complex clinical reports helps in finding valuable insights.

Figure 2 shows the 10V's of big data. Instead of the 10V's above, some suggest the following 5V's: Venue, Variability, Vocabulary, Vagueness, and Validity) [5].

To thrive in today's complex business environment, businesses must adopt a data-driven culture and leverage analytics platforms to make key decisions that improve productivity. Industries that benefit from big data include the healthcare, financial, airline, travel, restaurants, automobile, sports, agriculture, manufacturing, and hospitality industries. Big data technologies are playing an essential role in farming: machines are equipped with sensors that measure data in their environment.

\section{BIG DATA ANALYTICS}

Every day, data is growing bigger and bigger, and big data analysis (BDA) has become a requirement for gaining invaluable insights into data such that companies could gain significant profits in the global market. Once the big data is ready for analysis, we use advanced software programs such as Hadoop, MapReduce, MongoDB, and NoSQL databases [6]. Big data analytics refers to how we can extract, validate, translate, and utilize big data as a new currency of information transactions. It is an emerging field that is aimed at creating empirical predictions. Datadriven organizations use analytics to guide decisions at all levels [7].

Data scientists know how to use tools that identify patterns and relationships that may otherwise remain hidden. They are part of virtually every major industry, and manufacturing is no exception. Manufacturing big data analytics is expected to ensure better practices, decisionmaking, and a sustainable future for humankind. This will involve artificial intelligence and machine-learning technologies to determine better manufacturing practices and decision-making [8].

The manufacturer industry is beginning to deploy big data analytics for two essential tasks: gain control of the vast amounts of data generated and use the right information to drive productivity and enhance decision-making. Oneway manufacturers can take more control of their quality parameters is through data analytics. To stay competitive and keep pace with consumers' fickle buying habits, the manufacturing industry must consider implementing data analytics tools. The good thing about data analytics is that it can be implemented anywhere in the world.

\section{BIG DATA IN MANUFACUTING}

Data has long been the lifeblood of manufacturing. Manufacturing data is key to conducting data-driven manufacturing. Most manufacturing data is standardized which is supported by various industrial vendors and associations. Big data in manufacturing is generated from the production and process, from machines such as pumps, motors, compressors, meters, sensors, controllers or conveyers. In order to benefit and gain insight from the data collected, it must be analyzed and visualized. When big data is analyzed, it opens a window of opportunity for manufacturers to identify and fix problems before they get worse.

Six key drivers of big data applications in manufacturing have been identified as system integration, data, prediction, sustainability, resource sharing, and hardware. Based on the requirements of manufacturing, nine essential components of big data ecosystem have been identified as ingestion, storage, computing, analytics, visualization, management, workflow, infrastructure, and security [9]. Figure 3 shows big data in manufacturing [10].

\section{APPLICATIONS OF BIG DATA IN MANUFACTURING}

The data in manufacturing is often used for operational purposes manufacturing organization. Figure 4 shows how car manufacturers use big data [11]. Different applications of big data in manufacturing include [12]:

\section{- Preventive Maintenance}

Operational efficiency relies on the availability of the machinery in the production process. Manufacturers can use the best big data analytics software to determine the status of the machine. This approach is often called preventive maintenance.

\section{- Product Design}

Developing new products can be expensive. Big data analytics software can analyze data and help identify trends and market changes that can be fed into the design of new products.

\section{- Production Management Automation}

Automation of the production management is probably the hardest way of using big data in manufacturing processes. A good example of production management automation is the case with GE's wind turbines. Sensors provide data on energy generation and wind direction, according to which the blade pitch is changed to optimize the wind turbine's efficiency.

\section{- Customer Experience}

Excellent customer experience has become an essential part of every business. The big data analytics solutions draw customer data from a wide variety of sources and use the data for in-depth data analysis.

\section{- Supply Chain Improvement}

Timing is everything. Big data enables manufacturers to track the exact location of their products. Modern supply chains are becoming increasingly complex. Big data analytics solutions deliver supply chain visibility to instantly know key supply chain information. Big data can provide information about the supply chain and makes it possible to predict with greater certainty whether or not a supplier will deliver as agreed. It also increases transparency into the entire supply chain.

\section{BENEFITS}

With big data analytics, your company can improve manufacturing, customize product design, ensure better quality assurance, manage the supply chain, evaluate for any potential risk [13]. Big data analytics can help business to make informed and timely decisions, which are crucial for business improvement and growth. In a very competitive environment, manufacturers can turn to big data to improve their business operations. Big data can take a manufacturer from predictive decision making to prescriptive decision making.

Other benefits include $[12,14,15]$ :

- Reduced Process Flaws. Data analytics can greatly improve assembly-line efficiency. 
- Increasing Accuracy and Yield. Big data can improve product accuracy during the production process.

- Improving Quality Assurance. Many manufacturers have turned to big data to help them improve their quality assurance.

- Speeding Up Assembly. It helps manufacturing companies to know where they are most efficient, with the added possibility of producing more products in that area.

- Improved Workforce Efficiency. Big data in manufacturing can improve productivity, growth, and workforce efficiency.

- Competitive Advantage. It is becoming a crucial way for companies to outperform their peers by leveraging data-driven strategies.

- Increase Efficiency. Manufacturing big data can greatly improve production line speed and quality, which helps a manufacturing company control costs, increase productivity, and boost margins.

- Improved Customer Service. Big data analytics solutions can help manufacturers better gauge customer sentiment and respond to customers in real time.

- Regulatory Compliance. Data analytics can be effectively used to ensure regulatory compliance.

- Better forecasting. Predictive modeling helps in better forecasting of a host of business aspects.

\section{CHALLENGES}

As far as big data is concerned, the manufacturing industry has its share of challenges to contend with. The challenges include the following [14]:

- Complexity. Due to the complex nature of manufacturing adoption of latest technologies is always difficult.

- Environmental Issues. Something drastic needs to be done to reduce the adverse effects of manufacturing on the environment.

- Regulation Requirements. The manufacturing industry inadvertently exposes itself to safety risks (to humans) arising out of industrial accidents.

- Product Development \& Innovation. Manufacturers must constantly innovate in order to stay relevant and compete.

- Preventive Maintenance. Minimum disruption to business operations is one of the main challenging tasks for manufacturers.

- Faster Time-to-market. Manufacturers have a very short time-span within which to launch their products, else they risk losing out to competitors.

- Market Volatility. Market volatility constrains continuous product performance, thus impeding business performance.

- Huge Data \& Manual Analysis. Manual analysis is inherently time-consuming and risk-laden (human errors).

- Judicious Use. Big data often leads managers to rely too much on the data and abdicate decision making. They need to be judicious in their use of data especially around privacy and sensitivity concerns.

- Cyber Attacks. With the increasing adoption of emerging technologies in the manufacturing industry, the security concerns are becoming significant. Manufacturing is one of the most targeted industries by cyber attackers owing to the presence of vital data related to company and government, making manufacturers subjected to cybersecurity incidents.

\section{CONCLUSION}

The big data era has emerged. Use of data analytics to make decisions about business strategy and operations is becoming commonplace. Today, leveraging big data has become a business imperative since it is enabling solutions to long-standing business challenges for manufacturing companies worldwide. The top or leading manufacturers are poised to use big data technologies to capitalize on this and many other sources and expand their customer base across foreign countries. For those manufacturing businesses that are still wondering what big data can do for them, the sooner they get using big data analytics, the sooner it will be able to apply the latest innovations in data science. Once they do so, the sky's the limit. More information on big data in manufacturing can be found in the book in [16] and related journal: Journal of Big Data.

\section{REFERENCES}

[1] "Real-time applications of big data that drive various industries," https://elysiumpro.in/applications-big-data-driveindustries/

[2] "The 42 V's of big data and data science," https://www.kdnuggets.com/2017/04/42-vs-bigdata-data-science.html

[3] M. N.O. Sadiku, M. Tembely, and S.M. Musa," Big data: An introduction for engineers," Journal of Scientific and Engineering Research, vol. 3, no. 2, 2016, pp. 106-108.

[4] P. K. D. Pramanik, S. Pal, and M. Mukhopadhyay, "Healthcare big data: A comprehensive overview," in N. Bouchemal (ed.), Intelligent Systems for Healthcare Management and Delivery. IGI Global, chapter 4, 2019, pp. 72-100.

[5] J. Moorthy et al., "Big data: Prospects and challenges," The Journal for Decision Makers, vol. 40, no. 1, 2015, pp. 74-96.

https://www.grandviewresearch.com/industryanalysis/industrial-wireless-sensor-networks-iwsnmarket

[6] M. N. O. Sadiku, J. Foreman, and S. M. Musa, "Big data analytics: A primer," International Journal of Technologies and Management Research, vol. 5, no. 9, September 2018, pp. 44-49.

[7] C. M. M. Kotteti, M. N. O. Sadiku, and S. M. Musa, "Big data analytics," Invention Journal of Research Technology in Engineering \& Management, vol. 2, no. 10, Oct. 2018, pp. 2455-3689.

[8] M. Ryan, "Agricultural big data analytics and the ethics of power," Journal of Agricultural and Environmental Ethics, vol. 33, 2020, pp. 49-69.

[9] Y. Cui, S. Kara, and K. C.Chan, "Manufacturing big data ecosystem: A systematic literature review," Robotics and Computer-Integrated Manufacturing, vol. 62, April 2020 
[10] A. Bekker, "Big data in manufacturing: Use cases + Guide on how to start,"

https://www.scnsoft.com/blog/big-data-inmanufacturing-use-cases

[11] R. Delgado, "How car manufacturers are using big data," June 2015,

https://datafloq.com/read/car-manufacturersare-using-big-data/1204

[12] "Top 5 big data analytics software benefits for manufacturing in 2019," January 2019,

https://blogs.opentext.com/big-data-analyticsbenefits-manufacturing/

[13] L. Andrews, "What are examples of big data in manufacturing?" October 2019,

https://www.sensrtrx.com/what-are-examplesof-big-data-in-manufacturing/

[14] "Challenges of the manufacturing industry, \& big data analytics," August 2017,

https://www.msrcosmos.com/blog/challengesof-the-manufacturing-industry-big-data-analytics/

[15] R. Delgado, "Applications of big data in manufacturing," April 2016,

https://www.automation.com/en-

us/articles/2016-1/applications-of-big-data-inmanufacturing

[16] S. Khanna, Big Data In Manufacturing: A Practical Introductory Guide to Starting Your Big Data Journey. Kindle Edition,2017.

\section{ABOUT THE AUTHORS}

Matthew N.0. Sadiku is professor emeritus in the Department of Electrical and Computer Engineering at Prairie View A\&M University, Prairie View, Texas. He is the author of several books and papers. His areas of research interests include computational electromagnetics and computer networks. He is a fellow of IEEE.

Tolulope J. Ashaolu is a postdoctoral research fellow at Southwest University. He is the author of several papers and two books. His research interests include functional foods and food microbiology.

Abayomi Ajayi-Majebi is a professor in the Department of Manufacturing Engineering at Central State University in Wilberforce, Ohio. In 2015 he was honored by the White House as a Champion of Change for his significant contributions to the engineering education of minority students. He is a senior member of both the Society of Manufacturing Engineers and the American Society for Quality.

Sarhan M. Musa is a professor in the Department of Engineering Technology at Prairie View A\&M University, Texas. He has been the director of Prairie View Networking Academy, Texas, since 2004. He is an LTD Sprint and Boeing Welliver Fellow.

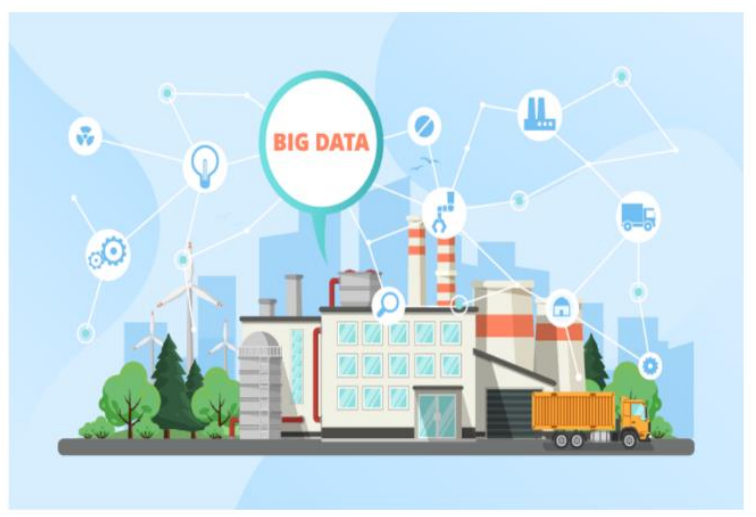

FIGURE 3: Big data in manufacturing [10].

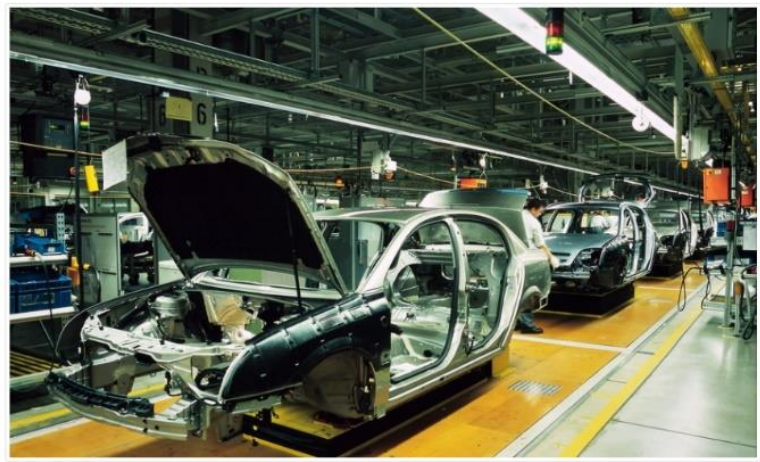

FIGURE 4: How car manufacturers are using big data [11]. 